\title{
A Unified Computational Approach to the Optimization of Surface Textures: One Dimensional Hydrodynamic Bearings
}

\author{
Agata Guzek ${ }^{*}$, Pawel Podsiadlo and Gwidon W. Stachowiak \\ Tribology Laboratory, School of Mechanical Engineering, The University of Western Australia \\ 35 Stirling Hwy, Crawley, Western Australia 6009, Australia \\ *Corresponding author: agata@mech.uwa.edu.au
}

( Manuscript received 20 April 2010; accepted 13 July 2010; published 31 July 2010 )

\begin{abstract}
In tribological applications, surface textures are used to increase load capacity and reduce friction losses in hydrodynamic lubricated contacts. However, there is no systematic, efficient and general approach allowing for the optimization of surface texture shapes to give an optimal performance. The work conducted is, in most cases, by "trial and error", i.e. changes are introduced and their effects studied. This is time consuming and inefficient. In this paper, a unified computational approach to the optimization of texture shapes in bearings is proposed. The approach aims at finding the optimal texture shape that supports the maximum load and/or minimizes friction losses in one dimensional hydrodynamic bearings. The texture shape optimization problem is transformed into a nonlinearly constrained mathematical programming problem with general constraints that can be solved using optimal control software. Load-carrying capacity or friction force of a bearing becomes an objective functional that is maximized or minimized, subject to: (i) any Reynolds equations given by first order ordinary differential equations, (ii) pressure boundary conditions and (iii) functions/parameters that define the surface texture shape. This newly developed approach is demonstrated on examples of parallel textured hydrodynamic bearings. The effects of non-Newtonian fluids, cavitation and viscosity varying with temperature are considered.
\end{abstract}

Keywords: hydrodynamic bearings, surface texture, geometric shapes, shape optimization

\section{Introduction}

Over decades numerous papers, both experimental and theoretical, have been published on textured surfaces $^{1-4)}$. Surface textures in the form of grooves, dimples, holes, chevrons and other shapes have been investigated for their possible reduction of friction and wear, increase of load-carrying capacity and avoidance of seizure and damage of contact surfaces.

One of the main problems is to find an optimal texture shape for contacting surfaces in hydrodynamic bearings that maximizes the load-carrying capacity for a given minimum film thickness and/or minimizes the friction force.

Various approaches were used in the texture shape optimization. Commonly used approach is based on exhaustive search. To perform the search, it is necessary to either manufacture bearings with various textured surfaces and conduct experiments or run a number of numerical simulations of Reynolds or Navier-Stokes equations for various textures. For the former, a laser surface texturing (LST) technology was used to produce microasperities on bearing surfaces and the effects of changes in geometry of the asperities on load and friction of mechanical seals ${ }^{5-7)}$, piston rings ${ }^{8-11)}$ and thrust bearings ${ }^{12,13)}$ were experimentally investigated. For the latter, numerical simulations were used to obtain pressure fields from Reynolds equations and load capacity and friction for parallel thrust bearings textured with square dimples of different depth and length ratios $^{14)}$ and spherical dimples of different radii and area ratios ${ }^{12)}$. In another study, two-dimensiona (2D) fluid flow between parallel walls was simulated using Navier-Stokes equations ${ }^{15)}$ and load capacity and friction were calculated for varying grove width and depth. However, the approach based on exhaustive search is time-consuming and the cost of performing many tests is often prohibitively high.

Another approach is analytical solutions, however, due to their complex nature, the shape optimization has been only achieved for one-dimensional (1D) cases and relatively simple $2 \mathrm{D}$ cases. The earliest attempts at the bearing optimization were made in 1918. Rayleigh 
showed, using the calculus of variations, that the optimal load capacity is achieved with a step bearing shape $^{16)}$. Rohde and McAllister found the optimal shape of a 1D journal bearing analytically ${ }^{17}$. They developed an optimization algorithm which was based on distributed control theory. Later, the algorithm was extended and used to optimize a $2 \mathrm{D}$ slider profile. For this optimization, first the film profile was represented as a distributed control in the principal coefficients of the Reynolds equation, and then load, which is the integral of the solution of this equation, was maximized $^{18)}$. Another variational calculus based method was developed by Maday ${ }^{19)}$ to account for an inequality constraint on the film thickness. Pontryagin's Maximum Principle was used to incorporate this constraint in optimization.

Recently, with the development of computational techniques and increasing processing power available, a substantial amount of work has been conducted using numerical methods. The shape optimization problem has been solved using nonlinear programming, heuristic algorithms (genetic algorithms and neural networks) and partial differential equation (PDE)-constrained optimization with the adjoint approach. Application of nonlinear programming methods was presented by Wang ${ }^{20)}$. A 2D lubrication model based on the Reynolds equation was analyzed to obtain the optimal film profiles for 2D elliptical and slider bearings. Unconstrained nonlinear programming techniques, such as a simplex method and a guided grid search (lattice search) were used. Buscaglia et al. ${ }^{21}$ ) optimized a 2D slider with the static surface covered with square microdimples. Size and depth of the microdimples were optimized using a genetic algorithm based on commercially available GENESIS code with the aim of minimizing the friction. Sinanoglu et al. ${ }^{22)}$ analyzed the pressure distribution in a journal bearing for various shaft surface textures, both experimentally and numerically for 1D flow governed by the Reynolds equation. For simulations, an artificial neural network designed by trial and error was used. A film height optimization in hydrodynamic slider bearings was undertaken by Ostayen et al. ${ }^{23)}$. The pressure distribution in a 2D slider bearing was described by the Reynolds equation. A PDE-constrained optimization solver was used with the application of the discrete adjoint approach for gradient calculation. In this way the optimal film height was obtained. However, the optimization was conducted for the film height distribution and not for the bearing texture shape.

Previous studies showed that the analytical and numerical methods work well for specific constraints imposed on pressure, film thickness, sliding ratio and lubricant rheology. This leads to difficulties when the shape optimization is conducted for new and/or complex, more realistic constraints. So far this problem has only been partially addressed by either developing new methods or modifying existing methods. However, this has resulted in a proliferation of various ways of optimizing surface shapes and difficulties associated with the selection of a right method. The only solution to this problem is a unified general approach that works for a wide range of constraints and arbitrary surface shapes.

In this paper, a unified computational approach to the optimization of $1 \mathrm{D}$ bearing surface texture shapes has been developed. This unified approach is based on a computational approach that was originally developed by Goh and $\mathrm{Teo}^{24,25)}$ for optimal control problems. The underlying idea is a transformation of the texture shape problem to a combined optimal control and parameter selection problem in such a way that the spacial variable is time, the load or friction is an objective functional to be optimized, subject to the Reynolds equations which are the system dynamics, pressure boundary conditions which are canonical constraints and texture shape parameters which are system parameters, control and state constraints. Using this transformation the shape optimization becomes a nonlinearly constrained mathematical programming problem that can be efficiently and accurately solved using optimal control software programs. The newly developed unified approach will be demonstrated on examples of 1D step, parallel and pad bearings. The optimal results will be compared against published data.

\section{Methods}

\subsection{Texture shape optimization (TSO)}

The optimization of texture shapes in $1 \mathrm{D}$ bearings can be stated as a nonlinearly constrained optimization problem in the following way:

Find the texture shape $h(x)$ that maximizes (or minimizes) a given objective functional $g(h(x))$

subject to the constraints that

- the Reynolds equation $R E(p(x), h(x))=0$ is satisfied, and

- boundary conditions that pressure vanishes at bearing edges $p(0)=p(L)=0$

where $g$ is an integral that represents a friction force $F=\int_{0}^{t} \tau(x) d x$ or a load $W=\int_{0}^{t} p(x) d x$ or other optimization objectives.

2.2. Combined optimal control and optimal parameter selection (COC-OPS)

TSO can be formulated as the following COC-OPS problem:

Maximize (or minimize) an objective functional

$$
G_{0}(u, z)=\phi_{0}\left(x\left(t_{f}\right), z\right)+\int_{t_{s}}^{t_{f}} g_{0}(t, x(t), u(t), z) d t
$$

over the control signal $u(t) \in R$ and the system parameters $z=\left[z_{1}, \ldots, z_{n_{z}}\right]^{\mathrm{T}} \in R^{n_{z}}$, subject to

- the system dynamics

$$
\dot{\boldsymbol{x}}(t)=\boldsymbol{f}(t, \boldsymbol{x}(t), u(t), \boldsymbol{z})
$$


- the initial conditions

$$
\boldsymbol{x}\left(t_{s}\right)=\boldsymbol{x}^{0}(\boldsymbol{z})
$$

- all-time control constraints

$$
\alpha_{k} u(t)+\beta_{k} \geq 0, \quad k=1,2, \ldots, n_{g l}
$$

- the canonical form constraints

$$
G_{k}(u, z)=\phi_{k}\left(x\left(\tau_{k}\right), z\right)+\int^{\tau_{k}} g_{k}(t, x(t), u(t), z) d t^{\geq} 0, k=1,2, \ldots, n_{g c}
$$

- the system parameter only constraints

$$
G_{k}(z)^{\geq} 0, \quad k=1,2, \ldots, n_{g z}
$$

where $\left\lfloor t_{s}, t_{f}\right\rfloor$ is the time interval, $\boldsymbol{x}(t)=\left[x_{l}(t), \ldots, x_{n_{s}}(t)\right]^{T} \in R^{n_{s}}$ is the state function vector,

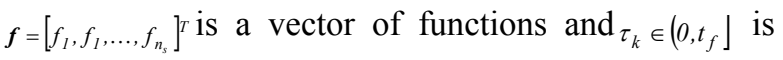
the characteristic time associated with the constraint $G_{k}$.

For the COC-OPS correspondences between the TSO and the optimal control are employed by replacing:

- the spacial variable with the time $(x=t)$,

- the film thickness with the control signal $(h(x)=$ $u(t))$,

- integration constants and film shape parameters with the system parameters,

- the load or friction with the objective functional,

- the Reynolds equation with the system dynamics, and

- the pressure boundary conditions with all-time control and canonical form constraints.

\subsection{Control parametrization}

Let the control signal be represented by a sum of basis functions with parameters $\left\{\sigma_{j}, j=1,2, \ldots, k\right\}^{24)}$

$$
u(t)=\sum_{j=1}^{k} \sigma_{j} B_{j}(t)
$$

where $\quad B_{j}(t)=\left\{\begin{array}{lr}1, & t_{j-1} \leq t \leq t_{j} \\ 0, & \text { otherwise }\end{array} \quad\right.$ is $\quad$ a piecewise constant function defined over a set of knots $\left\{t_{s}=t_{0}, t_{1}, \ldots, t_{k}=t_{f}\right\}$. Once the control signal is parametrized the objective functional and all the constraint functionals become functions of the parameter vector $\Theta=\left[\sigma_{1}, \ldots, \sigma_{k}, z_{1}, \ldots z_{n_{z}}\right]^{\mathrm{T}} \in R^{n_{p}} \quad$ and the COC-OPS problem becomes a nonlinear mathematical programming problem (NLMP), i.e.

$$
\underset{\Theta}{\operatorname{minimize}} \tilde{G}_{0}(\Theta)
$$

subject to the constraints

$$
\begin{aligned}
& \widetilde{G}_{i}(\Theta)=0, i=1,2, \ldots, n_{e} \\
& \widetilde{G}_{i}(\Theta) \geq 0, i=n_{e}+1, \ldots, n_{g c} \\
& u_{j}^{L}<\sigma_{j}<u_{j}^{U}, \quad j=1, \ldots, k
\end{aligned}
$$

$$
z_{k}^{L}<z_{k}<z_{k}^{L}, \quad k=1, \ldots, n_{z}
$$

where $u_{i j}^{L}, z_{k}^{L}$ and $u_{i j}^{U}, z_{k}^{L}$ are the lower and upper limits of the control signal and the system parameters, respectively.

Consequently, the TSO problem can be solved as a sequence of approximate problems $\left\{\operatorname{NLMP}_{p}\right\}_{p=1}^{\infty}$ in such a way that the solution of each approximate problem $\mathrm{NLMP}_{p}$ is a suboptimal solution of the COC-OPS problem. Convergence to an optimal solution would be deemed when subsequent reduction of functional values of $\tilde{G}_{0}(\Theta)$ is less than some prescribed threshold tolerance. This parametrized problem can be solved using Matlab software.

\section{Results}

Several examples of 1D hydrodynamic bearings were selected to illustrate the unified approach proposed in this work. Texture shapes were optimized for the maximum load capacity or the minimum friction force. The bearings selected satisfy the simplifying assumptions listed in Table 1. Convergence of the shape optimization process is assumed when the subsequent reduction in $\tilde{G}_{0}(\Theta)$ is less than $10^{-7}$. Cavitation effects were included as the Swift-Stieber condition at the vapor region in the film, i.e. $p=0$ if $p<0$ at the liquid-gas boundary.

Table 1 Summary of simplifying assumptions for one-dimensional bearings

\begin{tabular}{|l|l|}
\hline Assumptions & Comments \\
\hline 1 & $\begin{array}{l}\text { They are infinitely wide and the } \\
\text { film thickness is small compared } \\
\text { to the bearing length. }\end{array}$ \\
\hline 2 & $\begin{array}{l}\text { There is no variation of pressure } \\
\text { across the fluid film, i.e. } \\
\partial p / \partial y=0\end{array}$ \\
\hline 3 & The lubricant flow is laminar. \\
\hline 4 & $\begin{array}{l}\text { The inertial effect and the body } \\
\text { forces are negligible. }\end{array}$ \\
\hline 5 & $\begin{array}{l}\text { There is no slip at the bearing } \\
\text { surfaces. }\end{array}$ \\
\hline 6 & $\begin{array}{l}\text { There is no vertical flow across the } \\
\text { film. }\end{array}$ \\
\hline 7 & $\begin{array}{l}\text { The lubricant is incompressible } \\
\text { and isoviscous. }\end{array}$ \\
\hline
\end{tabular}

\#Example 1: Step bearing - load capacity optimization For a slider bearing with parallel surfaces maximize a load capacity with respect to an arbitrary shape film thickness $h^{*}\left(x^{*}\right)$

$$
\underset{h^{*}\left(x^{*}\right)}{\operatorname{maximize}} W=\int_{0}^{l} p^{*}\left(x^{*}\right) d x
$$

subject to

- the Reynolds equation 


$$
\frac{d p^{*}\left(x^{*}\right)}{d x^{*}}=\frac{h^{*}\left(x^{*}\right)-\bar{h}^{*}\left(x^{*}\right)}{h^{*}\left(x^{*}\right)^{3}}
$$

- $\quad$ the pressure boundary conditions

$$
p^{*}(0)=p^{*}(1)=0
$$

Using the unified approach the shape optim problem can be defined as follows

$$
\underset{u(t), z_{1}}{\operatorname{minimize}} G_{0}\left(u, z_{1}\right)=-\int_{0}^{1} x_{1}(t) d t
$$

subject to

- $\quad$ the system dynamics

$$
\frac{d x_{1}(t)}{d t}=\frac{u(t)-z_{1}}{u(t)^{3}}
$$

- $\quad$ the initial condition

$$
x_{1}(0)=0 \text {, and }
$$

- the canonical constraint

$$
G_{I}\left(u, z_{l}\right)=x_{l}(1)=0
$$

where $u(t)=h^{*}\left(x^{*}\right)$ and $z_{l}=\bar{h}\left(x^{*}\right)$

The optimal solution of this problem is a Rayleigh step slider bearing ${ }^{16)}$ shown in Fig. 1. If the coordinate $x^{*}$ is changed to a polar coordinate and the integration used for the calculation of load capacity is performed from 0 to $2 \pi$ the optimal solution will be a step journal bearing $^{19)}$.

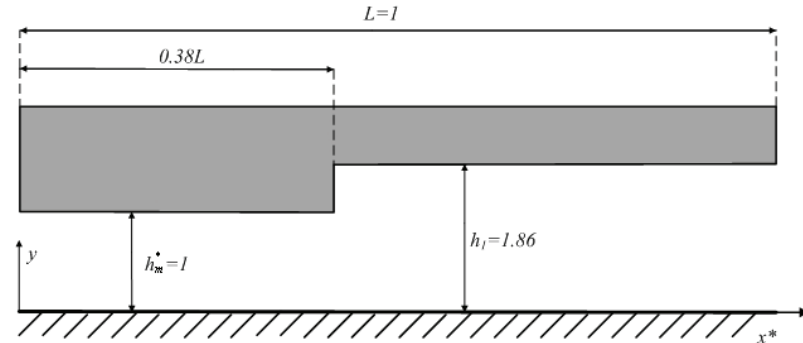

Fig. 1 A Rayleigh step bearing

\#Example 2: Partially textured parallel bearing with rectangular or elliptical dimples - load capacity optimization

For a parallel bearing with rectangular or elliptical dimples, shown in Fig. 2 and 3, respectively, maximize a load capacity with respect to dimple height and length ratios

$$
\underset{\varepsilon, \xi}{\operatorname{maximize} W}=\int_{0}^{l} p^{*}\left(x^{*}\right) d x
$$

subject to

- the Reynolds equation

$$
\frac{d p^{*}\left(x^{*}\right)}{d x^{*}}=\frac{h^{*}\left(x^{*}, \xi, \varepsilon\right)-\bar{h}^{*}\left(x^{*}\right)}{h^{*}\left(x^{*}, \xi, \varepsilon\right)^{3}}
$$

- $\quad$ the pressure boundary conditions

$$
p^{*}(0)=p^{*}(1)=0
$$

a. rectangular dimples (Fig. 2)

$$
h^{*}\left(x^{*}, \xi, \varepsilon\right)= \begin{cases}l_{0}<x^{*}<1-l_{1} \text { and } \\ \quad \bmod \left(x^{*}-l_{0}, \frac{D(1+\varepsilon)}{(m(1+\varepsilon)-1)}\right)<\varepsilon \frac{D}{(1+\varepsilon) m-1} \\ 1 \quad \text { otherwise }\end{cases}
$$

where $\zeta=h_{D} / h_{m}{ }^{*}$ is the dimple height ratio, and $\varepsilon=l_{D} / l$ is the dimple length ratio.

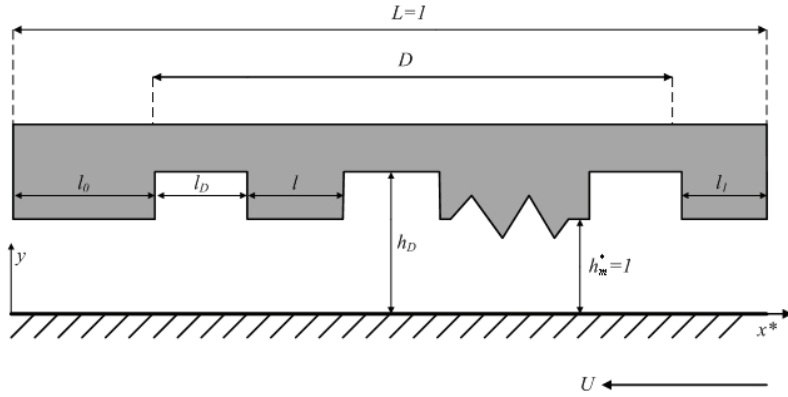

Fig. 2 Geometry of the partially textured parallel bearing with rectangular microdimples

b. elliptical dimples (Fig. 3)

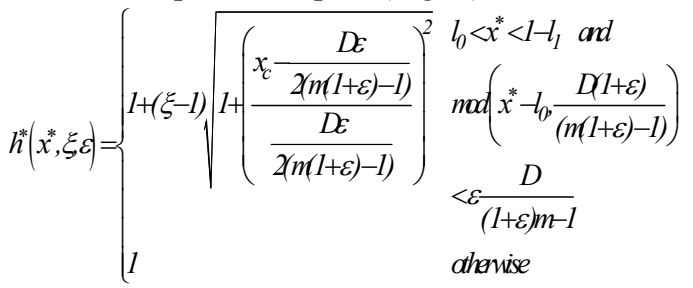

where $\zeta=h_{D} / h_{m}{ }^{*}$ is the dimple height ratio, $\varepsilon=2 r / l$ is the dimple length ratio,

$x_{c}=\bmod \left(x^{*}-l_{0}, D(1+\varepsilon) /(m(1+\varepsilon)-1)\right) \quad$ is the spacial coordinate defined at the starting point of each ellipse.

The optimization problem can be defined in a unified manner as follows:

$$
\underset{z_{1}, z_{2}, z_{3}}{\operatorname{minimize}} G_{0}\left(z_{1}, z_{2}, z_{3}\right)=-\int_{0}^{1} x_{1}(t) d t
$$

subject to

$$
\text { the system dynamics }
$$

$$
\frac{d x_{l}(t)}{d t}=\frac{h\left(t, z_{2}, z_{3}\right)-z_{l}}{h\left(t, z_{2}, z_{3}\right)^{3}}
$$

- the initial condition

$$
x_{l}(0)=0 \text {, and }
$$

- the canonical constraint

$$
G_{0}\left(z_{1}, z_{2}, z_{3}\right)=x_{1}(1)=0
$$

where, $z_{1}=\bar{h}\left(x^{*}\right), z_{2}=\zeta, z_{3}=\varepsilon$ and

a. rectangular dimples (Fig. 2)

$$
h^{*}\left(t, z_{2}, z_{3}\right)=\left\{\begin{array}{cc} 
& l_{0}<t<1-l_{1} \text { and } \\
z_{2} & \bmod \left(t-l_{0}, \frac{D\left(1+z_{3}\right)}{\left(m\left(1+z_{3}\right)-1\right)}\right)<z_{3} \frac{D}{\left(1+z_{3}\right) m-1} \\
& \text { otherwise }
\end{array}\right.
$$




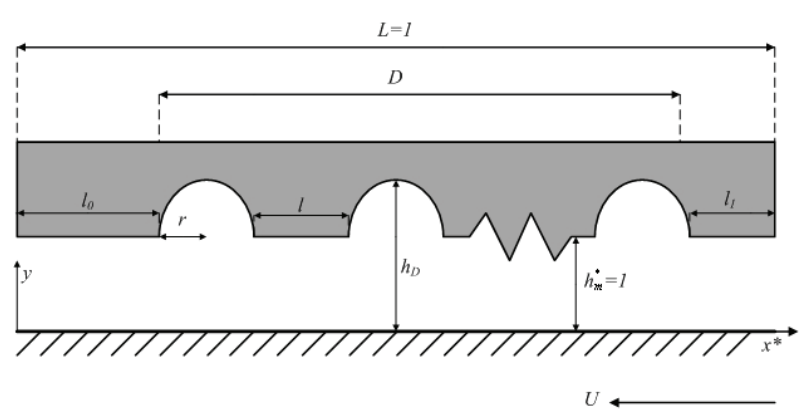

Fig. 3 Geometry of the partially textured parallel bearing with elliptical microdimples

A comparison of the optimization results for rectangular dimples with the results of the exhaustive search conducted by Rahmani et al. ${ }^{14)}$ is shown in Table 2 . Optimal length ratio was 1.00 for all numbers of dimples.

Table 2 Optimal height ratio and load obtained for parallel bearing with square dimples

\begin{tabular}{|l|l|l|l|}
\hline$m$ & \multicolumn{2}{|l|}{ Optimal height ratio $\xi$} & \multicolumn{1}{|}{$\begin{array}{c}\text { Optimal load } W^{*} \\
{\left[10^{-3}\right]}\end{array}$} \\
\hline & $\begin{array}{l}\text { Unified } \\
\text { approach }\end{array}$ & $\begin{array}{l}\text { Exhaustive } \\
\text { search [14] }\end{array}$ & Unified approach \\
\hline 2 & 1.567 & 1.568 & 8.947 \\
\hline 3 & 1.559 & 1.559 & 7.860 \\
\hline 5 & 1.554 & 1.555 & 7.166 \\
\hline 10 & 1.550 & 1.551 & 6.766 \\
\hline 25 & 1.549 & 1.549 & 6.552 \\
\hline
\end{tabular}

$$
\begin{aligned}
& \text { b. elliptical dimples (Fig. 3) }
\end{aligned}
$$

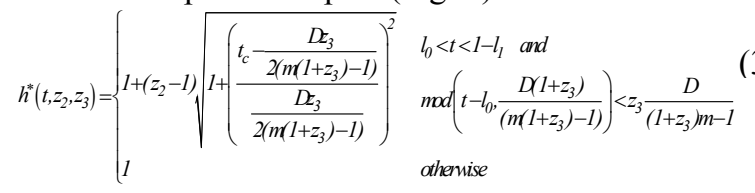

where $\left.t_{c}=x_{c}=\bmod \left(x-l_{0}, D\left(1+z_{3}\right) / m\left(1+z_{3}\right)-1\right)\right)$. A comparison between results obtained using the unified computational approach and the exhaustive search for elliptical dimples is shown in Table 3. Optimal length ratio was 1.00 for all numbers of dimples.

\#Example 3: Partially textured parallel bearing with elliptical dimples lubricated by non-Newtonian fluids load capacity optimization

For the bearing shown in Fig. 3 a non-Newtonian fluid behaviour, described by a Rabinowicz fluid model, was used, i.e.:

$$
\tau_{x y}+\kappa \tau_{x y}^{3}=\eta_{0} \frac{\partial u}{\partial y}
$$

where the nonlinear factor $\kappa<0$ is for the dilatant, $\kappa=$ 0 for Newtonian and $\kappa>0$ for pseudoplastic fluids, respectively.

The optimization aim is to maximize the load capacity with respect to the elliptical dimple height and length ratios (Fig. 3)
Table 3 Optimal height ratio and load obtained for parallel bearing with elliptical dimples

\begin{tabular}{|l|l|l|l|}
\hline$m$ & \multicolumn{2}{|l|}{ Optimal height ratio $\xi$} & $\begin{array}{l}\text { Optimal load } W^{*} \\
{\left[10^{-3}\right]}\end{array}$ \\
\hline & $\begin{array}{l}\text { Unified } \\
\text { approach }\end{array}$ & Numerical & Unified approach \\
\hline 2 & 1.720 & 1.730 & 8.534 \\
\hline 3 & 1.716 & 1.719 & 7.500 \\
\hline 5 & 1.710 & 1.712 & 6.839 \\
\hline 10 & 1.708 & 1.707 & 6.457 \\
\hline 25 & 1.706 & 1.705 & 6.255 \\
\hline
\end{tabular}

$$
\underset{\varepsilon, \xi}{\operatorname{maximize}} W=\int_{0}^{1} p^{*}\left(x^{*}\right) d x
$$

subject to

- the Reynolds equation under steady-state condition

$\frac{\partial}{\partial x^{*}}\left[h^{*}\left(x^{*}, \xi, \varepsilon\right)^{3} \frac{\partial p}{\partial x^{*}}+3 \alpha / 20^{*}\left(x^{*}, \xi, \varepsilon\right)^{5}\left(\frac{\partial p}{\partial x^{*}}\right)^{3}\right]=\frac{\partial h^{*}\left(x^{*}, \xi, \varepsilon\right)}{\partial x^{*}}$

- the pressure boundary conditions

$$
p^{*}(0)=p^{*}(1)=0
$$

where $p^{*}$ is the steady-state pressure, $\alpha$ is the dimensionless form of $\kappa(-1<\alpha<1 ; \alpha<0$ for dilatant, $\alpha=0$ for Newtonian, $\alpha>0$ for pseudoplastic fluids).

Since the integrated equation is difficult to solve analytically, the perturbation method of solving differential equations of higher orders was employed as described in .

By substituting $p^{*}=p^{*}{ }_{0}+\alpha p^{*}{ }_{1}$, in accordance with the perturbation method, the above equation becomes

$$
\begin{gathered}
\frac{\partial}{\partial x^{*}}\left[h^{*}\left(x^{*}, \xi, \varepsilon\right)^{3} \frac{\partial\left(p_{0}^{*}+\alpha p_{1}^{*}\right)}{\partial x^{*}}+\right. \\
\left.+3 \alpha / 2 h^{*}\left(x^{*}, \xi, \varepsilon\right)^{5} \frac{\partial\left(p_{0}^{*}+\alpha p_{l}^{*}\right)^{3}}{\partial x^{*}}\right]=\frac{\partial h^{*}\left(x^{*}, \xi, \varepsilon\right)}{\partial x^{*}}
\end{gathered}
$$

and the two following equations are obtained:

$$
\begin{gathered}
\frac{\partial}{\partial x^{*}}\left[h^{*}\left(x^{*}, \xi, \varepsilon\right)^{3} \frac{\partial p_{0}^{*}}{\partial x^{*}}\right]=\frac{\partial h^{*}\left(x^{*}, \xi, \varepsilon\right)}{\partial x^{*}} \\
\frac{\partial}{\partial x^{*}}\left[h^{*}\left(x^{*}, \xi, \varepsilon\right)^{3} \frac{\partial p_{1}^{*}}{\partial x^{*}}+3 / 20^{*}\left(x^{*}, \xi, \varepsilon\right)^{5}\left(\frac{\partial p_{0}^{*}}{\partial x^{*}}\right)^{3}\right]=0
\end{gathered}
$$

Therefore after integrating the equations:

$$
\begin{gathered}
\frac{\partial p_{0}^{*}}{\partial x^{*}}=\frac{h^{*}\left(x^{*}, \xi, \varepsilon\right)-c_{1}}{h^{*}\left(x^{*}, \xi, \varepsilon\right)^{3}} \\
\frac{\partial p_{1}^{*}}{\partial x^{*}}=\frac{-3 / 20^{*}\left(h^{*}\left(x^{*}, \xi, \varepsilon\right)-c_{1}\right)^{3}}{h^{*}\left(x^{*}, \xi, \varepsilon\right)^{7}}+\frac{c_{2}}{h^{*}\left(x^{*}, \xi, \varepsilon\right)^{3}}
\end{gathered}
$$

The optimization problem can be formulated in a unified manner as follows

$$
\underset{z_{1}, z_{2}, z_{3}, z_{4}}{\operatorname{minimize}} G_{0}\left(z_{1}, z_{2}, z_{, 3}, z_{4}\right)=-\int_{0}^{1}\left(x_{1}(t)+\alpha x_{2}(t)\right) d t
$$

subject to 
- $\quad$ the system dynamics

$$
\begin{gathered}
\dot{x}_{l}(t)=\frac{h^{*}\left(t, z_{2}, z_{3}\right)-z_{1}}{h^{*}\left(t, z_{2}, z_{3}\right)^{3}} \\
\dot{x}_{2}(t)=\frac{-\frac{3}{20}\left(h^{*}\left(t, z_{2}, z_{3}\right)-z_{1}\right)^{3}}{\left(h^{*}\left(t, z_{2}, z_{3}\right)\right)^{7}}+\frac{z_{4}}{h^{*}\left(t, z_{2}, z_{3}\right)^{3}}
\end{gathered}
$$

- $\quad$ the initial conditions

$$
x_{1}(0)=x_{2}(0)=0, \text { and }
$$

- $\quad$ the canonical constraints

$$
\begin{aligned}
& G_{1}\left(z_{1}, z_{2}, z_{3}, z_{4}\right)=x_{1}(1)=0 \\
& G_{2}\left(z_{1}, z_{2}, z_{3}, z_{4}\right)=x_{2}(1)=0
\end{aligned}
$$

where, $t=x^{*}, z_{1}=c_{1}, z_{2}=\zeta, z_{3}=\varepsilon, z_{4}=c_{2}$. The film thickness equation $h^{*}\left(t, z_{2}, z_{3}\right)$ is the same as in the example 2 .

Table 4 Optimal height ratio and load obtained for parallel bearing with 2 elliptical dimples lubricated by non-Newtonian fluids

\begin{tabular}{|l|l|l|}
\hline$\alpha$ & $\begin{array}{l}\text { Optimal height } \\
\text { ratio }\end{array}$ & Optimal load $W\left[10^{-3}\right]$ \\
\hline-0.9 & 1.731 & 8.582 \\
\hline-0.7 & 1.730 & 8.571 \\
\hline-0.5 & 1.729 & 8.561 \\
\hline-0.3 & 1.728 & 8.550 \\
\hline-0.1 & 1.727 & 8.540 \\
\hline 0 & 1.726 & 8.529 \\
\hline 0.1 & 1.725 & 8.519 \\
\hline 0.3 & 1.724 & 8.508 \\
\hline 0.5 & 1.724 & 8.498 \\
\hline 0.7 & 1.723 & 8.487 \\
\hline 0.9 & 1.723 & 8.484 \\
\hline
\end{tabular}

For the nonlinear factor $\alpha$ ranging from -0.9 to 0.9 in steps of 0.2 the maximum load $W_{\max }$ and the optimal dimple height ratio $\zeta$ were found. Length ratio was 1.00 for all cases. The results obtained for 2 dimples are shown in Table 4. Figure 4 illustrates the results for positive and negative alpha and for Newtonian fluid for different numbers of dimples. It was found that the load capacity was greater for dilatant fluids $(\alpha<0)$ than for Newtonian fluids. Performance of bearings lubricated with pseudoplastic fluids $(\alpha>0)$ was worse than that of bearings lubricated with Newtonian or dilatant fluids. These findings agree well with published data ${ }^{26)}$.

\#Example 4: Partially textured parallel bearing with elliptical dimples and lubricant viscosity changing with temperature - load capacity optimization

The parallel bearing with elliptical dimples was also optimized for lubricant viscosity changing with temperature. For simplicity, a linear model for temperature change ${ }^{27)}$ was chosen, i.e.

$$
T=\left(T_{1}-T_{0}\right) t-T_{0}
$$

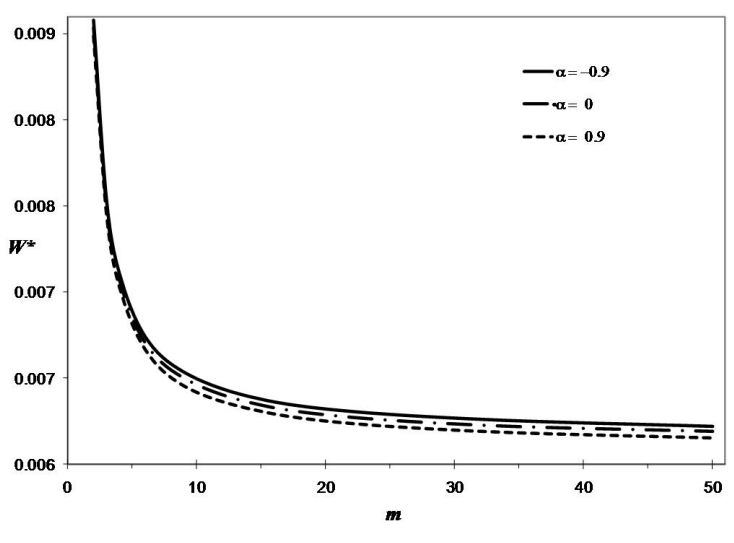

Fig. 4 Load versus the number of dimples for Newtonian $\alpha=0$, dilatant $\alpha<0$, and pseudoplastic, $\alpha>0$, fluids

where $T_{0}$ and $T_{1}$ are the temperatures at the bearing inlet and outlet, respectively. The change of viscosity with temperature is governed by the Vogel equation

$$
\eta^{*}=\frac{\eta}{\eta_{1}}=\frac{a}{\eta_{1}} e^{b /(T-c)}
$$

where chosen values of parameters were $\frac{a}{\eta_{l}}=0.0164$, $\mathrm{b}=300$ and $\mathrm{c}=220$. Temperatures chosen at the bearing inlet and outlet were $20{ }^{\circ} \mathrm{C}(293 \mathrm{~K})$ and $60{ }^{\circ} \mathrm{C}$ $(333 \mathrm{~K})$, respectively. For the temperature decrease the value of dimensionless viscosity, 1 at $20{ }^{\circ} \mathrm{C}$ dropped to 0.23 at $60{ }^{\circ} \mathrm{C}$ (about $80 \%$ ).

Table 5 Optimal height ratio and load obtained for parallel bearing with elliptical dimples and lubricant with constant viscosity and viscosity changing with temperature

\begin{tabular}{|l|l|l|l|l|}
\hline $\mathrm{m}$ & \multicolumn{2}{|l|}{$\begin{array}{l}\text { Viscosity changing } \\
\text { with temperature }\end{array}$} & \multicolumn{2}{|l|}{ Constant viscosity } \\
\hline & $\begin{array}{l}\text { Optimal } \\
\text { height } \\
\text { ratio }\end{array}$ & $\begin{array}{l}\text { Load } \\
{\left[10^{-3}\right]}\end{array}$ & $\begin{array}{l}\text { Optimal } \\
\text { height } \\
\text { ratio }\end{array}$ & $\begin{array}{l}\text { Load } \\
{\left[10^{-3}\right]}\end{array}$ \\
\hline 2 & 1.748 & 3.020 & 1.720 & 8.534 \\
\hline 3 & 1.737 & 2.566 & 1.716 & 7.500 \\
\hline 5 & 1.728 & 2.333 & 1.710 & 6.839 \\
\hline 10 & 1.723 & 2.185 & 1.708 & 6.457 \\
\hline 25 & 1.719 & 2.087 & 1.706 & 6.255 \\
\hline
\end{tabular}

Optimization results obtained for the bearing with changing viscosity are listed in Table 5 and shown in Fig. 5, and compared to a constant viscosity case. Length ratio was 1.00 for all numbers of dimples. The results showed that with the temperature increase the load capacity of the bearing decreases. This agrees with the fact that at higher temperatures the lubricant viscosity decreases and subsequently the lubricant capacity to support load is reduced.

\#Example 5: Partially textured parallel bearing with 
rectangular or elliptical dimples - friction force optimization

Although previous results were obtained only for load capacity, the unified approach proposed can be used for other optimization objectives, e.g. a minimal friction force or a maximal stiffness. In this example, an optimization in regards to minimizing the friction force will be presented. This optimization is possible as the objective functional $G_{0}(u, z)$ has a generic form.

For a parallel bearing with rectangular or elliptical dimples, shown in Fig. 2 and 3, respectively, minimize a friction force with respect to dimple height and length ratios

$$
\underset{\varepsilon, \xi}{\operatorname{minimize}} F=\int_{0}^{1} \frac{4 h-3 \bar{h}}{h^{2}} d x
$$

subject to

- the Reynolds equation

$$
\frac{d p^{*}\left(x^{*}\right)}{d x^{*}}=\frac{h^{*}\left(x^{*}, \xi, \varepsilon\right)-\bar{h}^{*}\left(x^{*}\right)}{h^{*}\left(x^{*}, \xi, \varepsilon\right)^{3}}
$$

- the pressure boundary conditions

$$
p^{*}(0)=p^{*}(1)=0
$$

a. rectangular dimples defined in Example 2a.

b. elliptical dimples defined in Example 2b.

The optimization problem can be defined in a unified manner as follows:

$$
\underset{z_{1}, z_{2}, z_{3}}{\operatorname{minimize}} G_{0}\left(z_{1}, z_{2}, z_{3}\right)=\int_{0}^{1} \frac{4 h(t)-3 \bar{h}}{h(t)^{2}} d t
$$

subject to

- $\quad$ the system dynamics

$$
\frac{d x_{1}(t)}{d t}=\frac{h\left(t, z_{2}, z_{3}\right)-z_{1}}{h\left(t, z_{2}, z_{3}\right)^{3}}
$$

- $\quad$ the initial condition

$$
x_{l}(0)=0 \text {, and }
$$

- the canonical constraint

$$
G_{0}\left(z_{1}, z_{2}, z_{3}\right)=x_{1}(1)=0
$$

where $z_{1}=\bar{h}\left(x^{*}\right), z_{2}=\zeta, z_{3}=\varepsilon$ and

a. $h^{*}\left(t, z_{2}, z_{3}\right)$ as defined in Example 2a.

b. $h^{*}\left(t, z_{2}, z_{3}\right)$ as defined in Example $2 b$.

Comparisons between results obtained using the unified computational approach and the exhaustive search for rectangular and elliptical dimples are shown in Tables 6 and 7. Optimal height ratios were 2.00 and optimal length ratios were 3.00 for all numbers of dimples.

\section{Discussion}

In this study, a unified computational approach was developed to optimize texture shapes in 1D hydrodynamic bearings. The bearings satisfy the assumptions listed in Table 1. Using the approach developed optimal texture shapes that support the maximum load were found for step bearing and parallel slider bearing textured with rectangular and elliptical dimples with Newtonian fluid. The optimal shapes of
Table 6 Optimal friction obtained for parallel bearing with square dimples

\begin{tabular}{|l|l|l|}
\hline$m$ & \multicolumn{2}{|c|}{ Optimal friction $F_{L}{ }^{*}$} \\
\hline & Unified approach & $\begin{array}{l}\text { Numerical } \\
\text { simulation }\end{array}$ \\
\hline 2 & 0.954 & 0.953 \\
\hline 3 & 0.957 & 0.956 \\
\hline 5 & 0.959 & 0.957 \\
\hline 10 & 0.963 & 0.959 \\
\hline 25 & 0.969 & 0.959 \\
\hline
\end{tabular}

Table 7 Optimal friction obtained for parallel bearing with elliptical dimples

\begin{tabular}{|l|l|l|}
\hline$m$ & \multicolumn{2}{|c|}{ Optimal friction $F_{L}{ }^{*}$} \\
\hline & Unified approach & $\begin{array}{l}\text { Numerical } \\
\text { simulation }\end{array}$ \\
\hline 2 & 0.955 & 0.955 \\
\hline 3 & 0.957 & 0.957 \\
\hline 5 & 0.959 & 0.959 \\
\hline 10 & 0.961 & 0.960 \\
\hline 25 & 0.961 & 0.961 \\
\hline
\end{tabular}

surface textures found for the bearings agree with those reported in literature ${ }^{12,14)}$. This indicates that the approach developed is accurate and applicable to the $1 \mathrm{D}$ bearings with arbitrary shape and parallel textured surfaces.

Optimal texture shapes were also found for the parallel slider bearing with elliptical dimples lubricated by non-Newtonian fluids. The Rabinowicz non-Newtonian fluid model was applied. However, there are different models describing the non-Newtonian behaviour of lubricant, for example, the power-law fluid model to account for non-Newtonian fluid behaviour ${ }^{28,29)}$ or the approach that involves solving two-dimensional momentum equations and a constitutive equation modelling non-Newtonian fluid behaviour based on the cross-film integrals of viscosity ${ }^{30,31)}$. Most of these models involve solving PDEs and they will be applied in the future work, after a 2D unified approach is developed.

Optimization of texture shapes was also performed for parallel slider bearing with elliptical dimples lubricated by Newtonian fluids with temperature-dependant viscosity. For this optimization, a linear model for the lubricant temperature change was employed. However, more realistic and accurate temperature models ${ }^{32,33)}$ exist and they can be considered in future applications. To achieve this, the unified approach proposed has to be extended into PDEs. This is because the temperature distribution used in the models is calculated using the energy equation which has partial derivatives with respect to two independent variables, i.e. $x$ and $y$ coordinates.

Optimal square and elliptical dimples were obtained using the unified approach proposed, but other texture shapes can also be optimized, e.g. the side length of 


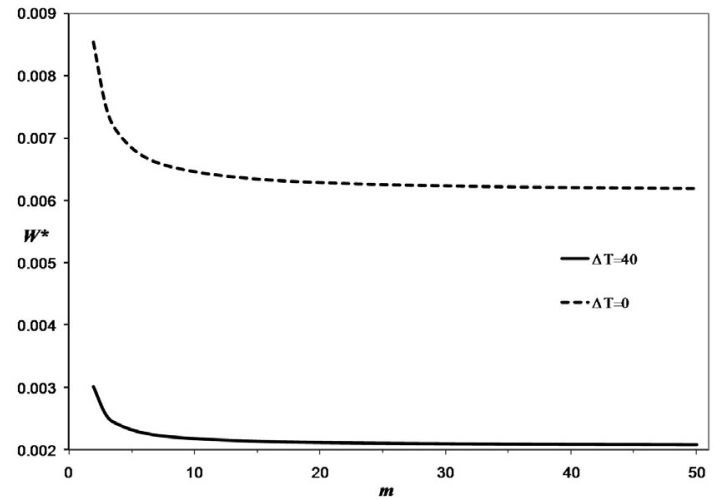

Fig. 5 Load versus the number of dimples considering dependence of viscosity on temperature

triangle dimple $\mathrm{e}^{34)}$.

In this work, the lubricant flow and the pressure between a stationary textured surface and a moving slider were described by the 1D Reynolds equations without taking into account inertia forces. These forces, however, can significantly affect the hydrodynamic lift, especially if the Reynolds number is large and the dimple texture aspect ratio (i.e. the ratio between the length of the dimple and its depth) is small ${ }^{35)}$. To include these effects a special form of the Reynolds equation ${ }^{32)}$ can be used in the unified approach.

In this study, cavitation effects were included in the Reynolds equation using the Swift-Stiebel condition, i.e. by not allowing the pressure to fall below 0 . However, more accurate cavitation models could be applied, as shown through an analysis of the influence of different cavitation models on the performance of microtextured journal bearings ${ }^{36)}$. It was found that the Reynolds model greatly underestimates the cavitation area, thereby overestimating the calculated friction torque. It was concluded that instead of this model, mass-conserving models should rather be applied, for instance with the use of so-called mass conserving Jakobsson-Floberg-Olsson (JFO) boundary conditions $^{37)}$.

One of possible ways to include these conditions is through the use of the Elrod and Adams model in the $p-$ $\theta$ formulation $^{38)}$. Studies showed that this model is able to predict accurately the film re-formation occurring at the texture-cell scale ${ }^{36)}$. Another approach used for mass-conserving cavitation calculations involves rewriting boundary value problem consisting of the Reynolds equation and the JFO boundary conditions as an arbitrary Langrangian-Eulerian (ALE) problem $^{39}$. Then, a finite element discretization of the equations can be performed and simulation results can be obtained. The method does not involve any discontinuities or switch functions, but it makes it necessary to solve two field equations (Reynolds equation and Laplace equation). Both models proposed above involve finite element and differential quadrature techniques, which require spacial discretization and forming a grid for computations. Therefore, they are unsuitable for use with the current 1D implementation of the unified computational approach. It is planned, however, to extend the unified approach to include a finite element method solver, which would enable incorporating mass-conserving cavitation models into calculations.

Incompressible flow between surfaces was assumed in this study. However, the unified approach developed can also be applied to bearings lubricated by compressible fluid. For example, the surface texture shape optimization can be performed for gas bearings described by the Reynolds equation with a Burgdorfer's correction for thin air gaps ${ }^{40)}$ or the Reynolds equation for a hydrostatic compressible flow over a single dimple $\mathrm{e}^{41,42)}$

In this study, the lubricant flow was assumed to be laminar. However, the flow is often turbulent (for the Reynolds numbers $>1000$ ) especially in high speed bearings. The Reynolds averaged Navier-Stokes equations can be used to describe turbulent flows for roughened surfaces ${ }^{43,44)}$. The unified approach will also be extended into PDEs in order to include turbulent flow effects.

The optimization results obtained for the 1D cases are helpful in understanding the relationships between various texture shapes and bearing performance. However, when designing optimal texture shapes for actual bearings an entire different category of problems is encountered as contacting surfaces are 2D objects. Subsequently, the optimization of surface texture shapes in 2Ds will involve solving PDEs. Thus, our future work will focus on the development of the unified approach for bearings with fluid flow governed by 2D Reynolds or Navier-Stokes equations.

\section{Conclusions}

From the work conducted the following conclusions can be drawn:

- A unified computational approach has been developed for the texture shape optimization of 1D hydrodynamic bearings. Unlike other approaches it allows for the optimization of texture shapes for a wide range of bearings, including step, journal, and parallel textured bearings. The approach works for bearings with fluid flow governed by the Reynolds equation that can be written in the form of $1 \mathrm{st}$ order differential equations. Arbitrary texture shapes and any texture shapes with geometry defined by a set of parameters can be optimized for the maximum load capacity, the minimum friction force and other optimization objectives.

- Although initial results were presented for bearings that satisfied the simplifying assumptions, given in Table 1, the approach can be modified (as already shown on some examples) in such a way that thermal, cavitation, inertia and turbulent flow effects, and compressible fluids can be included. Generally, this requires an extension of the 
approach into bearings with fluid flow governed by partial differential equations (energy equations and Navier-Stokes equations).

The ultimate goal of this work is to develop a unified computational approach for the optimization of 3D hydrodynamic and elastohydrodynamic lubrication contacts for any geometry of texture shapes and any lubricant rheology. The initial work conducted on 1D bearings is the first step towards this development.

\section{Acknowledgements}

The authors would like to thank the School of Mechanical Engineering, University of Western Australia for its support during the writing of this paper. Agata Guzek wishes to thank the Commonwealth Government of Australia for the award of an IPRS scholarship and the University of Western Australia for the award of an UPAIS scholarship.

\section{References}

[1] Moore, D. F., "A History of Research on Surface Texture Effects," Wear, 13, 1969, 381-412.

[2] Costa, H. L. and Hutchings, I. M., "Hydrodynamic Lubrication of Textured Steel Surfaces under Reciprocating Sliding Conditions," Tribology International, 40, 2007, 1227-1238.

[3] Etsion, I., "State of the Art in Laser Surface Texturing," ASME Journal of Tribology, 127, 1, 2005, 248-253.

[4] Li, Y. and Menon, A. K., "The Development and Implementation of Discrete Texture for the Improvement of Tribological Performance," ASME Journal of Tribology, 117, 2, 1995, 279-284.

[5] Etsion, I. and Burstein, L., "A Model for Mechanical Seals with Regular Microsurface Structure," Tribology Transactions, 39, 3, 1996, 677-683.

[6] Etsion, I. and Halperin, G., "A Laser Surface Textured Hydrostatic Mechanical Seal," ASLE Transactions, 45, 3, 2002, 430-434.

[7] Etsion, I., Kligerman, Y. and Halperin, G., "Analytical and Experimental Investigation of Laser-Textured Mechanical Seal Faces," Tribology Transactions, 42, 3, 1999, 511-516.

[8] Ryk, G., Kligerman, Y. and Etsion, I., "Experimental Investigation of Laser Surface Texturing for Reciprocating Automotive Components," ASLE Transactions, 45, 4, 2002, 444-449.

[9] Ryk, G., Kligerman, Y. and Shinkarenko, A., "Experimental Investigation of Partial Laser Surface Texturing for Piston-Ring Friction Reduction," Tribology Transactions, 48, 4, 2005 , 583-588.
[10] Ryk, G. and Etsion, I., "Testing Piston Rings with Partial Laser Surface Texturing for Friction Reduction," Wear, 261, 7-8, 2006, 792-796.

[11] Kligerman, Y., Etsion, I. and Shinkarenko, A., "Improving Tribological Performance of Piston Rings by Partial Surface Texturing," ASME Journal of Tribology, 127,3, 2005, 632-638.

[12] Brizmer, V., Kligerman, Y. and Etsion, I., "A Laser Surface Textured Parallel Thrust Bearing," Tribology Transactions, 46, 3, 2003, 397-403.

[13] Etsion, I., "Experimental Investigation of Laser Surface Textured Parallel Thrust Bearings," Tribology Letters, 17, 2, 2004, 295-300.

[14] Rahmani, R., Shirvani, A. and Shirvani, H., "Optimization of Partially Textured Parallel Thrust Bearings with Square-Shaped Micro-Dimples," Tribology Transactions, 50, 3, 2007, 401-406.

[15] Sahlin, F., Glavatskih, S. D., Almqvist, T. and Larsson, L., "Two-Dimensional CFD-Analysis of Micro-Patterned Surfaces in Hydrodynamic Lubrication," ASME Journal of Tribology, 127, 1, 2005, 96-102.

[16] Rayleigh, O. M., "Notes on the Theory of Lubrication," Philosophical Magazine, 35, 1, 1918, $1-12$.

[17] McAllister, G. T. and Rohde, S. M., "Optimum Design of One-Dimensional Journal Bearings," Journal of Optimization Theory and Applications, 41, 4, 1983, 599-617.

[18] Rohde, S. M. and McAllister, G. T., "On the Optimization of Fluid Film Bearings," Proceedings of the Royal Society of London Series A - Mathematical Physical and Engineering Sciences, 351, 1667, 1976, 481-497.

[19] Maday, C. J., "The Maximum Principle Approach to the Optimum One-Dimensional Journal Bearing," ASME Journal of Lubrication Technology, 92, 3, 1970, 482-489.

[20] Wang, N., "Engineering Optimum Design of Fluid-Film Lubricated Bearings," Tribology Transactions, 43, 3, 2000, 377-386.

[21] Buscaglia, G. C., Ciuperca, I. and Jai, M., "On the Optimization of Surface Textures for Lubricated Contacts," Journal of Mathematical Analysis and Applications, 335, 2, 2007, 1309-1327.

[22] Sinanoglu, C., Nair, F. and Baki Karamis, M., "Effects of Shaft Surface Texture on Journal Bearing Pressure Distribution," Journal of Materials Processing Technology, 168, 2, 2005 , 344-353.

[23] Van Ostayen, R. A. J., Van Beek, A. and Munnig-Schmidt, R., "Film Height Optimization of Hydrodynamic Slider Bearings," Proc. ASME/STLE International Joint Tribology Conference IJTC2007, San Diego, October 2007, 237-239.

[24] Goh, C. J. and Teo, K. L., "Control Parametrization: A Unified Approach to Optimal 
Control Problems with General Constraints," Automatica, 24, 1, 1988, 3-18.

[25] Teo, K. L., Goh, C. J. and Wong, K. H., "A Unified Computational Approach to Optimal Control Problems," Longman Scientific \& Technical, Harlow, Essex, 1991, 99-122.

[26] Lin, J. R., "Non-Newtonian Effects on the Dynamic Characteristics of One-Dimensional Slider Bearings: Rabinowitsch Fluid Model," Tribology Letters, 10, 4, 2001, 237-243.

[27] Stachowiak, G. W. and Batchelor, A., "Engineering Tribology," Elsevier Butterworth-Heinemann, Oxford, 2005, 175-176.

[28] Buckholz, R. H., "Effects of Power-Law, Non-Newtonian Lubricants on Load Capacity and Friction for Plane Slider Bearings," ASME Journal of Tribology, 108, 1, 1986, 86-91.

[29] Khlifi, M. E., Souchet, D., Hajjam, M. and Bouyahia, F., "Numerical Modeling of Non-Newtonian Fluids in Slider Bearings and Channel Thermohydrodynamic Flow," ASME Journal of Tribology, 129, 3, 2007, 695-699.

[30] Paranjpe, R. S., "Analysis of Non-Newtonian Effects in Dynamically Loaded Finite Journal Bearings Including Mass Conserving Cavitation," ASME Journal of Tribology, 114, 4, 1992, 736-744.

[31] Gecim, B., "Non-Newtonian Effects of Multigrade Oils on Journal Bearing Performance," Tribology Ttransactions, 33, 3, 1990, 384-394.

[32] Dobrica, M. B. and Fillon, M., "Thermohydrodynamic Behavior of a Slider Pocket Bearing," ASME Journal of Tribology, 128, 2006, 312-318.

[33] Dobrica, M. and Fillon, M., "Reynolds' Model Suitability in Simulating Rayleigh Step Bearing Thermohydrodynamic Problems," Tribology Transactions, 48, 2005, 522-530.

[34] Yu, H., Wang, X., and Zhou,F., "Geometric Shape Effects of Surface Texture on the Generation of Hydrodynamic Pressure between Conformal Contacting Surfaces," Tribology Letters, 37, 2010, 123-130.

[35] Dobrica, M. B. and Fillon, M., "About the Validity of Reynolds Equation and Inertia Effects in Textured Sliders of Infinite Width," Proceedings

\section{APPENDIX}

\section{List of symbols}

Hydrodynamic lubrication:

$h \quad$ film thickness, called texture shap

$h_{m} \quad$ minimum film thickness

$h^{*} \quad$ dimensionless film thickness, $h / h_{m}$

$h_{m}{ }^{*} \quad$ dimensionless minimum film thickness, $h_{m}{ }^{*}=1$

$\bar{h}^{*} \quad$ film thickness at maximum pressure of the Institute of Mechanical Engineers Part J: Journal of Engineering Tribology, 223, 2009, 69-78.

[36] Ausas, R. F., Ragot, P., Leiva, J., Jai, M., Bayada, G. and Buscaglia, G. C., "The Impact of the Cavitation Model in the Analysis of Microtextured Lubricated Journal Bearings," ASME Journal of Tribology, 129, 2007, 868-875.

[37] Folberg, L. J. and Jakobsson, B., "The Finite Journal Bearing, Considering Vaporization," Charmers Tekniska Hoegskolas Handlinger, 190, 1957, 1-116.

[38] Elrod, H. G. and Adams, M., "A Computer Program for Cavitation and Starvation Problems," Proc. First LEEDS-LYON Symposium on Cavitation and Related Phenomena in Lubrication. Leeds, UK, 1974, 37-41.

[39] Schweizer, B., "Numerical Approach for Solving Reynolds Equation with JFO Boundary Conditions Incorporating ALE Techniques," ASME Journal of Tribology, 131, 1, 2009, 011702.

[40] Jai, M., Buscaglia, G. C. and Iodanoff, I., "Multi-Constrained Optimization of Running Characteristics of Mechanisms Lubricated with Compressible Fluid," ASME Journal of Tribology, 126, 2004, 132-136.

[41] Van Odyck, D. E. A. and Venner, C. H., "Compressible Stokes Flow in Thin Films," ASME Journal of Tribology, 125, 2003, 543-551.

[42] Feldman, Y., Kligerman, Y. and Etsion, I., "The Validity of the Reynolds Equation in Modeling Hydrostatic Effects in Gas Lubricated Textured Parallel Surfaces," ASME Journal of Tribology, 128, 2006, 345-350.

[43] Villasmil, L. A., Chen, H. C. and Childs, D. W., "Evaluation of Near Wall Turbulence Models for Deliberately Roughened Liquid Annular Seals," AIAA Journal, 43, 2003, 2137-2146.

[44] Billy, F., Arghir, M. and Pineau, G., "Navier-Stokes Analysis of a Regular Two-Dimensional Roughness Pattern under Turbulent Flow Regime," ASME Journal of Tribology, 128, 2006, 122-130.

$\begin{array}{ll}h_{1} & \text { maximum film thickness } \\ p & \text { hydrodynamic pressure } \\ U & \text { runner velocity } \\ \eta & \text { fluid viscosity } \\ \eta_{0} & \text { viscosity of a Newtonian fluid } \\ \kappa & \text { nonlinear factor } \\ \alpha & \text { dimensionless nonlinear factor, } \\ & \alpha=\frac{36 \kappa \mu_{0}^{2} U^{2}}{h_{0}^{2}} \\ L^{*} & \text { bearing length } \\ p^{*} & \begin{array}{l}\text { dimensionless pressure under steady state, } \\ \end{array}\end{array}$


$p_{0}{ }^{*} \quad$ first component of the pressure $p^{*}$

$p_{1}{ }^{*} \quad$ second component of the pressure $p^{*}$

$\tau \quad$ shear stress

$W \quad$ dimensionless load carrying capacity

$F \quad$ dimensionless friction force

$x, y \quad$ Cartesian coordinates

$x^{*}$

$u$

$h_{D}$

$\zeta$

$l_{D}$

$l$

$\varepsilon$

$l_{0}$

$l_{1}$

$r$

$x_{c}$

point of an ellipse

$D \quad$ textured portion, $1-l_{0}-l_{1}$

$m \quad$ number of dimples

$T \quad$ bearing temperature

$T_{0} \quad$ temperature at the bearing inlet

$T_{1} \quad$ temperature at the bearing outlet

$\eta^{*} \quad$ dimensionless lubricant viscosity, $\eta^{*}=\frac{\eta}{\eta_{1}}$

$\eta_{1} \quad$ lubricant viscosity at the temperature $T_{0}$

$\frac{a}{\eta_{l}}, b, c$ given constants in the Vogel equation,

$$
\frac{a}{\eta_{1}}=0.0164, b=300, c=220
$$

$T \quad$ bearing temperature

$T_{0} \quad$ temperature at the bearing inlet

$T_{1} \quad$ temperature at the bearing outlet

Optimal control:

$t$ time

$u \quad$ control signal

$x \quad$ state variable

$x^{0} \quad$ initial conditions of $x$

$z \quad$ system parameters

$G_{0} \quad$ objective functional

$G_{k} \quad$ constraints functional

$f \quad$ system dynamics

$\tau_{k} \quad$ characteristic time

$t_{s} \quad$ starting time

$t_{f} \quad$ final time

$\alpha_{k}, \beta_{k} \quad$ scalar parameters

$g^{0}, g^{k} \quad$ scalar functions

$\varphi_{0}, \varphi_{k} \quad$ scalar functions

$n_{z} \quad$ number of system parameters

$n_{s} \quad$ number of state variables

$n_{g c} \quad$ number of canonical constraints

$n_{g l} \quad$ number of all-time control constraints

$n_{g z} \quad$ number of system parameter only constraints

$c_{1}, c_{2} \quad$ integration constants 